INPLASY

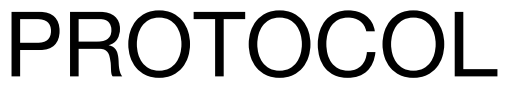

To cite: Zhang et al. Different surgical procedures for the treatment of patent ductus arteriosus in children: a protocol for a network metaanalysis. Inplasy protocol 2020110067. doi:

10.37766/inplasy2020.11.0067

Received: 16 November 2020

Published: 17 November 2020

Corresponding author:

Tao You

youtao2016@126.com

Author Affiliation:

Department of Cardiovascular

Surgery, Gansu Provincial

Hospital

Support: GSWSKY2016-04.

Review Stage at time of this submission: Preliminary searches.

Conflicts of interest:

There have no conflicts of interest to this work.

\section{Different surgical procedures for the treatment of patent ductus arteriosus in children: a protocol for a network meta-analysis}

Zhang, X1; Hou, XD2; Wang, WX33 YI, K4; Wang, X5; Ding, F6; $\mathrm{Li}, \mathrm{XX}^{7}$; You, $\mathrm{T}^{8}$.

Review question / Objective: P: Children younger than 18 years of age with patent ductus arteriosus I: Treatment of patent ductus arteriosus C: surgical ligation, fluoroscopy guided technique of percutaneous closure, fluoroscopy combined with TTE guided technique of percutaneous closure, TEE guided technique of perpulmonary device closure, TTE guided percutaneous closure of femoral artery and TTE guided percutaneous closure of femoral vein for PDA. $O$ the efficacy and safety of different surgical procedures for the treatment of patent ductus arteriosus.

Condition being studied: Patent ductus arteriosus in children. We will perform a comprehensive literature search until January 2021 in the following databases: PubMed, EMBASE, the Cochrane Library, Web of Science, and MEDLINE. The reference lists of articles and relevant systematic reviews will be tracked to identify other relevant studies.

INPLASY registration number: This protocol was registered with the International Platform of Registered Systematic Review and Meta-Analysis Protocols (INPLASY) on 17 November 2020 and was last updated on 17 November 2020 (registration number INPLASY2020110067).

\section{INTRODUCTION}

Review question / Objective: P: Children younger than 18 years of age with patent ductus arteriosus I: Treatment of patent ductus arteriosus C: surgical ligation, fluoroscopy guided technique of percutaneous closure, fluoroscopy combined with TTE guided technique of percutaneous closure, TEE guided technique of perpulmonary device closure, TTE guided percutaneous closure of femoral artery and TTE guided percutaneous closure of femoral vein for PDA. $O$ the efficacy and safety of different 
surgical procedures for the treatment of patent ductus arteriosus.

Condition being studied: Patent ductus arteriosus in children. We will perform a comprehensive literature search until January 2021 in the following databases: PubMed, EMBASE, the Cochrane Library, Web of Science, and MEDLINE. The reference lists of articles and relevant systematic reviews will be tracked to identify other relevant studies.

\section{METHODS}

Participant or population: Children younger than 18 years of age with patent ductus arteriosus.

Intervention: Treatment of patent ductus arteriosus.

Comparator: Surgical ligation, fluoroscopy guided technique of percutaneous closure, fluoroscopy combined with TTE guided technique of percutaneous closure , TEE guided technique of perpulmonary device closure, TTE guided percutaneous closure of femoral artery and TTE guided percutaneous closure of femoral vein for PDA.

Study designs to be included: Randomized controlled trials, prospective or retrospective cohort studies.

Eligibility criteria: 1.Types of study: We will include randomized controlled trials, prospective or retrospective cohort studies that reported the efficacy and safety of one of the 6 methods for the treatment of PDA. The number of cases included in each group must be greater than 10. Relevant systematic reviews or meta-analyses will be also included to track their references. 2. Participants: Children younger than 18 years of age with patent ductus arteriosus confirmed by clinical and transthoracic echocardiographic and scheduled for surgical ligation, percutaneous closure. 3. Interventions. surgical ligation, fluoroscopy guided technique of percutaneous closure, fluoroscopy combined with TTE guided technique of percutaneous closure, TEE guided technique of perpulmonary device closure, TTE guided percutaneous closure of femoral artery and TTE guided percutaneous closure of femoral vein for PDA or one of these 6 methods combines with another one. 4. Outcomes. The primary outcomes will include surgical success rate, operation time, intensive care unit (ICU) stay, Intraoperative radiation dose, radiation exposure time, total postoperative complication rate, postoperative major complication rate, any residual shunt after procedure. The secondary outcomes are total hospital stay, postoperative hospital stay, and total cost. 5. Other criteria. For similar studies published by the same author or institution, an article with a long follow-up or a larger number of studies will be included.

Information sources: PubMed, EMBASE, the Cochrane Library, Web of Science, and MEDLINE.

Main outcome(s): The efficacy and safety of different surgical procedures for the treatment of patent ductus arteriosus.

Quality assessment / Risk of bias analysis: The quality of all non-random studies will be assessed using (ROBINS-I) to assess the risk of bias of the included prospective or retrospective cohort studies. The risk of bias will be ranked as low, moderate, serious, critical risk of bias and no information. The quality of all RCTs will be assessed using the Cochrane Handbook version 5.1.0 to assess its risk of bias, and we will evaluate the risk of bias as low, high or unclear. The risk of bias assessment will be completed by two independent reviewers, and conflicts will be resolved by a third reviewer. Quality of evidence will be graded separately for each outcome using a tool suggested by the Grading of Recommendations Assessment, Development and Evaluation (GRADE) Working Group. The GRADE approach will assess five domains including study limitations, consistency of effect, imprecision, indirectness and publication bias to assess the quality of the body of evidence for each outcome. It is classified 
into 4 levels: high level, moderate level, low level, and very low level.

Strategy of data synthesis: Using Review Manager 5.3 (The Nordic Cochrane Centre, The Cochrane Collaboration, Copenhagen, Denmark) statistical analysis software, using 12 test and $x^{2}$ test to analyze the statistical heterogeneity of the included literature, when $P \geq 0.1$ and 12 When $\leq 50 \%$, the heterogeneity is considered acceptable, and the fixed-effects model is used for the combined analysis; if $\mathrm{P} 50 \%$, the heterogeneity is considered to be large, and the random effects model is used for analysis, and one by one Sensitivity analysis is performed by excluding literature. Binary data uses relative risk (RR) as the effect size, and continuous data uses the standardized mean difference (SMD) as the effect size, and the interval estimation uses $95 \%$ confidence intervals (95\% Cl). Network meta-analysis will be conducted using a Bayesian Markov chain Monte Carlo (MCMC) framework. We will use the deviance information criterion (DIC) to compare model fit and parsimony. The convergence will be assessed using the Brooks-Gelman-Rubin (BGR) plots method. The node splitting method will be used to examine the inconsistency between direct and indirect comparisons if a loop connecting 3 or more arms exist. If nodesplitting analysis determined $P<0.05$, the inconsistency model will be used for pooled analysis. Otherwise, the consistency model will be used. Besides, rank probabilities will be calculated, which indicate the probability for each treatment to be best, second best and so on. The analyses will be performed using $\mathbf{R}$ (version 3.4.1; R Foundation for Statistical Computing, Vienna, Austria) software.

Subgroup analysis: If the necessary data are available, subgroup analyses will be done for different types of participants by age, gender, and country.

Sensibility analysis: We will perform a sensitivity analysis to verify the robustness of the study results. This will be achieved by assessing the impact of the sample size, high risk of bias, missing data, and selected models. Following the analyses, if the quality of a study is judged to be low, it will be removed to ensure the robustness of the results.

Country(ies) involved: China.

Keywords: Patent ductus arteriosus, child, network meta-analysis, percutaneous closure, surgical ligation.

Contributions of each author:

Author 1 - Xin Zhang.

Author 2 - XiaoDong Hou.

Author 3 - WenXin Wang.

Author 4 - Kang YI.

Author 5 - Xinkuan Wang.

Author 6 - Fan Ding.

Author 7 - XinXin Li.

Author 8 - Tao You. 